\title{
Divertículo Solitário de Ceco Perfurado: Relato de Caso e Revisão da Literatura
}

\author{
Solitary Diverticulum Perforated of the Cecum: Report of Case and \\ Review of Literature
}

\author{
MÁRIO HENRIQUE LEITE DE ALENCAR ${ }^{1}$; MANLIO BASÍLIO SPERANZINI ${ }^{2}$; TIAGO SALESSI LINS ${ }^{3}$; \\ ALOÍSIO LAURINDO DE MENDONÇA ${ }^{4}$; CYOMARA SANCHES ATTAB ${ }^{5}$
}

\begin{abstract}
1. Cirurgião Oncológico; Membro Adjunto do Colégio Brasileiro de Cirurgiões; ${ }^{2}$ Professor Titular de Cirurgia do Aparelho Digestivo da Faculdade de Medicina do ABC - SP; Chefe do Serviço de Cirurgia Geral do Conjunto Hospitalar do Mandaqui, São Paulo - SP; ${ }^{3}$ Residente em Coloproctologia no Hospital Barão Lucena, Recife - PE;

4. Cirurgião Plástico e Cirurgião Geral do Serviço de Emergência do Conjunto Hospitalar do Mandaqui, São Paulo SP; ${ }^{5}$ Médica-Residente em Medicina Nuclear do Hospital de Câncer de Barretos e Ex-Plantonista do Pronto Socorro Clínico do Conjunto Hospitalar do Mandaqui, São Paulo - SP.
\end{abstract}

\begin{abstract}
ALENCAR MHL; SPERANZINI MB; LINS TS; MENDONÇA AL; ATTAB CS. Divertículo Solitário de Ceco Perfurado: Relato de Caso e Revisão da Literatura. Rev bras Coloproct, 2009;29(2): 242-245.

RESUMO: Divertículo solitário do intestino grosso é uma entidade relativamente rara, sendo mais frequentemente encontrado sob a forma de múltiplos divertículos. Tais divertículos são mais comuns no ceco e cólon ascendente, e geralmente são divertículos verdadeiros, ou seja, possuem todas as paredes do intestino. Quando o divertículo de ceco evolui para inflamação os pacientes geralmente se apresentam com sintomas sugestivos de apendicite aguda. Um caso de divertículo de ceco perfurado é relatado em uma mulher de 49 anos, cujo diagnóstico foi realizado somente após a cirurgia. Aspectos clínicos, propedêutica e terapia cirúrgica são discutidos.
\end{abstract}

Descritores: Divertículo; Diverticulite; Ceco; Apendicite; Cirurgia.

\section{INTRODUCÃO}

Divertículo solitário de ceco é uma entidade relativamente rara, mas que deve ser lembrada no diagnóstico diferencial das desordens da área ileocecal. Foi descrito pela primeira vez por Potier, 1912, em uma mulher de 32 anos. Desde então foram relatados apenas casos esporádicos de divertículo de ceco como causas de abdome agudo ${ }^{(1,2,3,4)}$.

\section{RELATO DE CASO}

Uma mulher branca, 49 anos, foi admitida no setor de emergência com história de cinco dias de dor abdominal progressiva, no qual havia iniciado como desconforto epigástrico com posterior irradiação para fossa ilíaca direita. Estava acompanhada de náuseas, porém sem vômitos. Alegava ainda o uso de Sulfametoxazol/ Trimetoprima e ácido acetilssalicílico por três dias sem prescrição médica. Negava febre, anorexia, sintomas dispépticos, perda de peso e alteração de história ginecológica. Sem antecedentes de doenças infecciosas, cirurgias e neoplasia familiar. Seu exame físico revelou regular estado geral, temperatura $37.2^{\circ} \mathrm{C}$, pulso de $88 \mathrm{bpm}$. Havia uma leve tensão abdominal, principalmente na fossa ilíaca direita e especialmente à palpação profunda. Sinal de Blumberg positivo com plastrão palpável. Sua contagem de células bran-

Trabalho realizado no Conjunto Hospitalar do Mandaqui - São Paulo. Departamento de Cirurgia Geral.

$\overline{\text { Recebido em 25/02/2008 }}$

Aceito para publicação em 17/03/2008 
cas foi de 15.500 com leve desvio à esquerda (2\% bastonetes). As radiografias de tórax e abdome estavam normais. Com hipótese diagnóstica de apendicite aguda foi proposta terapia cirúrgica. Realizado incisão de McBurney e no inventário da cavidade havia bloqueio da parede anterior do ceco por alças de intestino delgado que depois de desfeitas revelaram divertículo de ceco perfurado, com fecalito impactado em seu óstio interno (figura 1), e apêndice cecal normal. O divertículo media aproximadamente $5.0 \mathrm{~cm}$ no maior diâmetro e estava há $2.5 \mathrm{~cm}$ da válvula ileocecal. A tática operatória consistiu em apendicectomia propriamente dita, e diverticulectomia com reparo em dois planos ( $1^{\circ}$ total com vicryl 3.0 e seromuscular com prolene 3.0) e drenagem da cavidade (figura 2).

Seguiu-se curso de antibióticoterapia endovenosa com ciprofloxacino $800 \mathrm{mg} / \mathrm{dia}$ e metronidazol 1.5g/dia. Paciente evoluiu com íleo adinâmico por quatro dias com resolução espontânea. $\mathrm{O}$ dreno foi retirado no $5^{\circ}$ dia e a paciente recebeu alta assintomática no $7^{\circ}$ dia de pós-operatório. O estudo anátomopatológico revelou divertículo verdadeiro ulcerado com processo inflamatório agudo e serosite fibrinoleucocitária. $\mathrm{O}$ apêndice cecal era normal.

\section{DISCUSSÃO}

Em geral, os divertículos colônicos respeitam a seguinte distribuição anatômica; Cólons descendente e sigmoide com $80 \%$, cólon transverso $10 \%, 4 \%$ nos cólons ascendente e reto e $2 \%$ no ceco ${ }^{(5)}$. O divertículo solitário de ceco ocorre em menos de 2,5\% dos casos ${ }^{(6,7)}$ e pode estar associado à diverticulose generalizada em $5 \%{ }^{(8)}$. Em estudo de meta-análise não foi encontrada diferença significativa de incidência entre os sexos ${ }^{(9,10,11,12)}$.

Os divertículos do cólon podem ser classificados em dois grupos; verdadeiros ou congênitos e falsos ou adquiridos. Os divertículos verdadeiros possuem todas as paredes do cólon. Geralmente são únicos e ocorrem mais, frequentemente, no ceco e cólon ascendente. A tração do ceco em seu ponto de adesão ou a eversão da parede do ceco entre duas bandas de constrição (órgão peritonizado), durante a sua formação, seriam fatores etiológicos ${ }^{(7,13)}$. Hughes foi o primeiro autor a contestar sobre a origem congênita dos divertículos de ceco. Em sua série de 200 autópsias todos os divertículos de ceco (12\%) eram falsos divertículos. Divertículos falsos ou adquiridos são for-

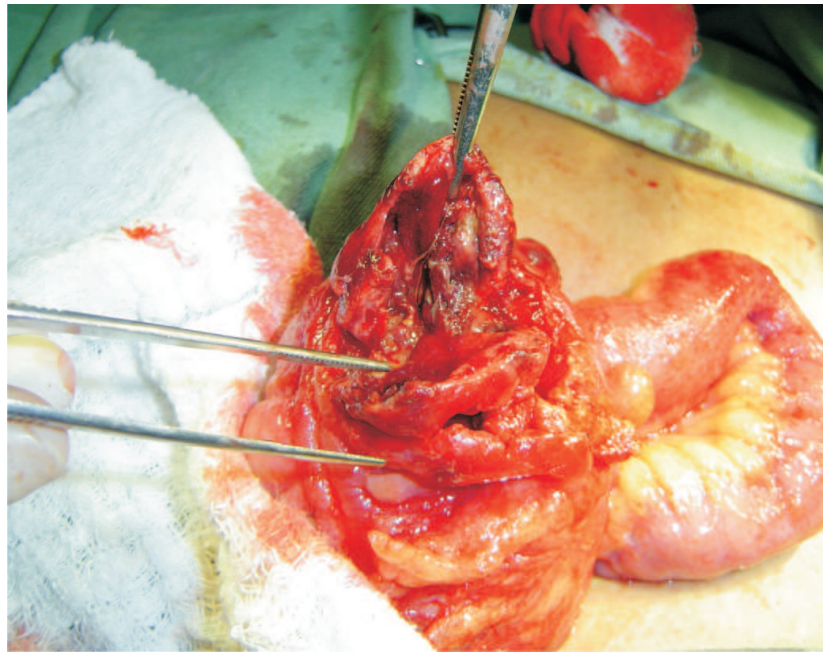

Figura 1 - Divertículo de ceco aberto, cirurgicamente, demonstrando no seu interior fecalito impactado.

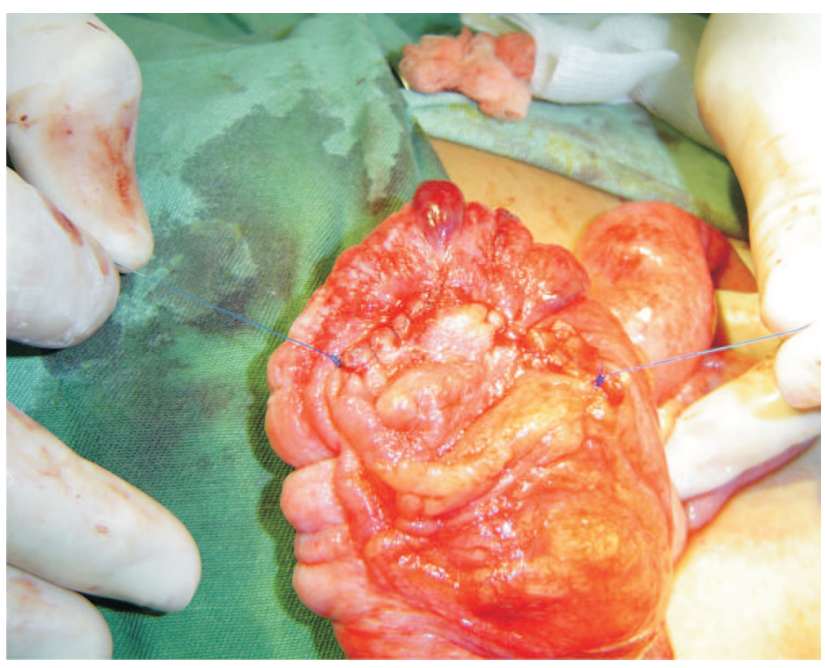

Figura 2 - Aspecto final do ceco, após correção cirúrgica (diverticulectomia).

mados pela herniação da mucosa e submucosa, através de um ponto de fragilidade da parede muscular. Usualmente, são múltiplos e ocorrem com mais frequência no cólon esquerdo, mas podem ocorrer em qualquer sítio do cólon. Aproximadamente $76 \%$ dos divertículos solitários do ceco situam-se dentro de uma área de $5 \mathrm{~cm}$ ao redor da válvula ileocecal. Este fato na opinião de alguns autores ${ }^{(6,9,14)}$ poderia explicar a patogênese do divertículo solitário de ceco.

Partindo do princípio de que haveria uma fragilidade congênita na parede do ceco, o alimento ao atravessar a válvula ileocecal colide com essa região, assumindo uma forma diverticular, durante o fechamento da válvula. A incidência da doença diverticular é maior 
em países industrializados e em regiões com baixa ingesta de fibras alimentares. O cólon depende da presença de uma quantidade mínima de massa luminar para propelir as fezes eficazmente no sentido do reto. Quando a quantidade de massa fecal é sub-ideal, as contrações segmentares geram uma pressão excessiva, causando herniações da mucosa, através de pontos de menor resistência da parede do cólon ${ }^{(15,16)}$.

As complicações ocorrem, devido à obstrução do óstio do divertículo por impactação fecal e resíduos alimentares, causando inflamação (mais comum), sangramento por erosão da parede dos vasos que penetram no divertículo (rara) e perfuração com formação de abscessos e fístula ${ }^{(6,7)}$. O processo inflamatório pode variar desde uma ulceração catarral até perfuração, levando a uma peritonite generalizada ou abscesso localizado. Verifica-se esta última complicação, sobretudo se a perfuração evolui de maneira lenta permitindo aderência com vísceras contíguas e parede abdominal ${ }^{(17)}$. A hemorragia diverticular proveniente do cólon direito é infrequente e geralmente não é grave ${ }^{(18,19)}$.

Quando o divertículo se manifesta de maneira crônica pode assumir a forma de um pseudotumor inflamatório. $\mathrm{O}$ exame objetivo da região pode demonstrar uma massa dolorosa, alongada e de consistência amolecida diferenciando-a de um tumor ${ }^{(20)}$. Porém a dúvida diagnóstica pode persistir, mesmo após a intervenção cirúrgica, quando então somente o estudo anátomopatológico poderá fazer o diagnóstico de certeza. O pseudotumor inflamatório pode se complicar, futuramente, com estenose do lúmem da víscera e espessamento da parede até a oclusão mecânica total, que também pode se dar por aderência, ou volvo pela retração cicatricial do tecido inflamado.

O divertículo do intestino grosso pode ser classificado em três categorias: 1. Assintomático, 2. Sintomático, 3. Complicado. Quando o divertículo solitário do ceco evolui para inflamação, geralmente, o paciente apresenta-se com sintomas sugestivos de apendicite aguda; dor na fossa ilíaca direita, aumento da tensão e contratura da parede abdominal, febre, leucocitose, raramente náusea e vômito. $\mathrm{O}$ diagnóstico diferencial inclui: Apendicite aguda, Doença inflamatória intestinal, Neoplasia maligna, Diverticulite de Meckel, Colite granulomatosa entre outros. Raramente, o diagnóstico correto é feito no pré-operatório. A hipótese diagnóstica realizada com maior frequência, nestes casos, é de apendicite aguda ${ }^{(18,21)}$.
A escolha do procedimento cirúrgico depende das condições locais, e por isso deve ser individualizado para cada paciente. Revisando a literatura, nota-se certa inconsistência a cerca do tratamento para esta patologia. A grande dúvida que existe entre os autores é quanto à radicalidade (hemicolectomia) ou não (diverticulectomia, drenagem e antibióticoterapia) da terapia a ser empregada. Em algumas séries de casos, a taxa de mortalidade para diverticulecotomia variou de $1,6 \%$ a $5 \%$, enquanto que para hemicolectomia em torno de $10 \%$ aproximadamente ${ }^{(14,22)}$. Em outras séries a taxa de mortalidade para simples drenagem e antibióticoterapia, variou de 0 a $0.9 \%{ }^{\left({ }^{(9)}\right.}$.

A hemicolectomia deve ser considerada como plano de tratamento nos seguintes casos; pacientes que apresentam extenso acometimento do ceco e cólon ascendente; incapacidade durante o ato cirúrgico de excluir carcinoma, pacientes com hemorragia digestiva baixa, cujo sítio exato não tenha sido detectado durante a cirurgia, porém com estudos pré-operatórios, demonstrando tratar-se de origem no cólon direito, situações em que o fechamento do defeito possa prejudicar a patência do lúmem intestinal, e por fím, quando a massa comprometer a válvula ileocecal, sendo esta última situação contestada por ser plenamente solucionada por ressecção econômica ${ }^{(23)}$. Quando a inflamação está limitada ao divertículo, uma simples diverticulectomia com inversão do coto pode ser feita tal como na apendicectomia.

Abscesso, após ruptura do divertículo, é tratado com drenagem local e exérese do divertículo. Se a parede do ceco estiver comprometida, uma tiflectomia parcial deve ser realizada caso a válvula ileocecal não esteja comprometida. A apendicectomia deverá ser realizada sempre que possível no mesmo tempo da diverticulectomia $^{(24)}$.

\section{CONCLUSÃO}

O caso relatado demonstra a dificuldade em se fazer o diagnóstico correto, antes da cirurgia. Por ser uma patologia de resolução, eminentemente, cirúrgica, apesar de rara, deve fazer parte das desordens da região ileocecal no diagnóstico diferencial. Devido à semelhança desta lesão com neoplasia maligna, a sua confirmação está diretamente relacionada com a extensão ou radicalidade da cirurgia. É recomendado apendicectomia no mesmo tempo cirúrgico da diverticulectomia, quando esta for factível. 
ABSTRACT: Solitary diverticulum of the large intestine is an entity relatively rare, being more frequently found under the form of multiple diverticulum. Such diverticulum's are more common in the cecum and ascending colon and they are usually true diverticulum, in other words, they possess all of the walls of the intestine. When the cecum diverticulum develops for inflammation the patients come usually with suggestive symptoms of acute appendicitis. A case of diverticulum perforated of the cecum is related in a 49 year-old woman, whose diagnosis was only accomplished after the surgery. Clinical aspects, propedeutic and surgical therapy are discussed.

Key words: Diverticulum; Diverticulosis; Cecum; Appendicitis; Surgery.

\section{REFERÊNCIAS}

1. Mayo CW. Diverticulitis of colon. Postgrad Med. 1950;8:368-73.

2. Greaney EM, Snyder WH. Acute diverticulitis of the cecum encountered at emergency surgery. Am J Surg. 1957;94:270-9.

3. Anderson L. Acute diverticulitis of the cecum. Study of ninetynine surgical cases. Surgery. 1947;22:479-88.

4. Remonda G, Caruso ML, Pugnani M. Solitary diverticulum of the caecum: case report. Ann Osp Maria Vittoria Torino. 1980;23:164-73.

5. Wagner DE, Zollinger RW. Diverticulitis of the cecum and ascending colon. Arch Surg. 1961;83:436-43.

6. Sardi A, Gokli A, Singer JA. Diverticular disease of the cecum and ascending colon. Am Surg. 1987;53:41-5.

7. Williams KL. Acute solitary ulcers and acute diverticulitis of the cecum and ascending colon. Br J Surg. 1960;47:351-8.

8. Hughes LE. Postmortem survey of diverticular disease of the colon. GUT. 1969;10:336-51.

9. Cutajar CL. Solitary cecal diverticula. Dis Colon Rectum. 1978;21:627-9.

10. Anscombe AR, Keddie NC, Schofield PF. Solitary ulcers and diverticulitis of the caecum. Br J Surg. 1967;4:553.

11. Kaufmann T, Forman J, Rojas R. Solitary diverticulum of the cecum. NY State J Med. 1986;86:598-9.

12. Parker RA, Serjeant JC. Acute solitary ulcer and diverticulitis of the caecum. Br J Surg. 1957;45:19.

13. Greensfelder LA, Hillier RI. Caecal diverticulosis with special reference to traumatic diverticula. Surg Gynecol Obstet. 1929;48:786-95.

14. Lauridsen J, Ross FP. Acute diverticulitis of the cecum. Arch Surg. 1952;64:320-30.
15. Painter NS, Truelove SC, Ardran GM, Tuckey M. Segmentation and the localization of intraluminal pressures in the human colon, with special reference to the pathogenesis of colonic diverticula. Gastroenterology. 1967;49:169-77.

16. Rolandelli RH, Roslyn JJ. Cólon e reto. In: Towsend CM, Beauchamp RD, Evers BM, editores. Sabiston Tratado de Cirurgia. $16^{\circ}$ ed. Rio de Janeiro: Guanabara Koogan; 2003. p.1036-9.

17. Dexter C J. Acute diverticulitis of the cecum with perforation. Am J Surg. 1955;90:535.

18. Galassi G. Note di terapia chirurgica delle complicazioni della malattia diverticolare del colon. Arch Atti Soc Ital Chir. 1977;2:223.

19. Podesta MT, Pace JL. Diverticular disease in Malta. St Luke's Josp Gaz Malta. 1973;8:126.

20. Alluminio P, Margarita V, Morozo G, Della Beffa V. Diverticolite acuta solitaria del cieco. Minerva Chir. 1987;42:975-6.

21. Waite VC. Diverticulitis of the cecum. Am J Surg. 1954;88:718.

22. Rodkey GV, Hermann G. Diverticulitis of the right colon. Am J Surg. 1961;101:61-5.

23. Schuler JG, Bayler J. Diverticulitis of the cecum. Surg Gynecol Obstet. 1983;156:743-8.

24. Bergman BB. The treatment of perforated diverticulum of the cecum. Int Surg. 1968;50:447-449.

Endereço para correspondência: MÁRIO HENRIQUE LEITE DE ALENCAR

Rua 48, 221, Bairro Jardim Alvorada.

14780-210 - Barretos, SP

Tel.: (17) 33236912

E-mail: marinho.alencar@terra.com.br 